\title{
Pancreatic Stone Extracorporeal Shockwave Lithotripsy-A New Concern for Urologists?
}

\author{
Nitin Sharma ${ }^{1}$, Jay A. Motola ${ }^{2}$ \\ ${ }^{1}$ Department of Urology, Lenox Hill Hospital, New York, NY (NS) \\ ${ }^{2}$ Department of Urology, Mount SInai-Roosevelt, New York, NY (JAM)
}

*Corresponding Author: Nitin Sharma, MD, Department of Urology, Lenox Hill Hospital, New York, NY (NS), USA, Email: NitinSharmaMD@gmail.com

\begin{abstract}
Extracorporeal shock wave lithotripsy (ESWL) has been adopted by multiple medical disciplines as an effective and relatively safe modality for stone fragmentation. Complication rates are typically low but can vary from those with minimal consequence to life-threatening injuries. We report the first case of a perinephric hematoma with resulting acute kidney injury (AKI) following an intended pancreatic stone ESWL. We believe that this complication resulting from inaccurate lithotripter stone targeting of the pancreas can be prevented in the future with improved preoperative patient assessment and early urologic management in the setting of concurrent renal stones and renal pathology.
\end{abstract}

Purpose: Urologists are not familiar with pancreatic lithotripsy nor are gastroenterologists familiar with the side effects of renal lithotripsy. We present a case highlighting both of these entities.

Materials and Methods: A case of pancreatic lithotripsy is presented with the unusual complication of a perinephric hematoma.

Results: A potentially serious complication arising from an intended pancreatic lithotripsy occurred as a result of inadvertent targeting of a pancreatic stone.

Conclusions: With careful planning and collaboration between specialties, urologic complications of pancreatic lithotripsy can be avoided.

Keywords: Pancreatic lithotripsy, subcapsular hematoma, hydronephrosis.

Abbreviations: $E S W L=$ extracorporeal shockwave lithotripsy, $A K I=$ acute kidney injury, $E R C P=$ endoscopic cholangiopancreatography, $U P J=$ ureteropelvic junction, $R P O=$ right posterior oblique, $C T=$ computerized tomography

\section{INTRODUCTION}

Extracorporeal shock wave lithotripsy (ESWL) has become an effective treatment option for pancreatic stones since its first use in 1987[1]. Indications for ESWL for pancreatic stones are generally reserved for large impacted stones within the pancreatic duct or stones proximal to ductal strictures. ESWL therapy is often offered to fragment such stones prior to endoscopic removal with stone-free rates as high as $70-85 \%$ [2]. However, this non-invasive modality for treating pancreatic stone disease is not without its complications.

Similar to its use in urinary lithiasis, ESWL treatment can result in complications to adjacent organs owing to the blast effects of its shockwaves. In the case of pancreatic stone
ESWL, complications such as acute pancreatitis, hepatic subcapsular hematoma and splenic injuries are well-documented in the literature [35]. We report the first case of a perinephric and subcapsular hematoma with AKI following pancreatic stone ESWL.

\section{CASe Report}

A 78-year-old African American female with history of chronic pancreatitis and multiple pancreatic duct stones was evaluated by the Department of Gastroenterology. The patient had multiple endoscopic cholangiopancreatographies (ERCP) in the past with incomplete resolution of a large stone burden (Figure 1) and persistent pain attributable to pancreatic disease. Pancreatic ESWL therapy was subsequently offered for further staged stone fragmentation. 


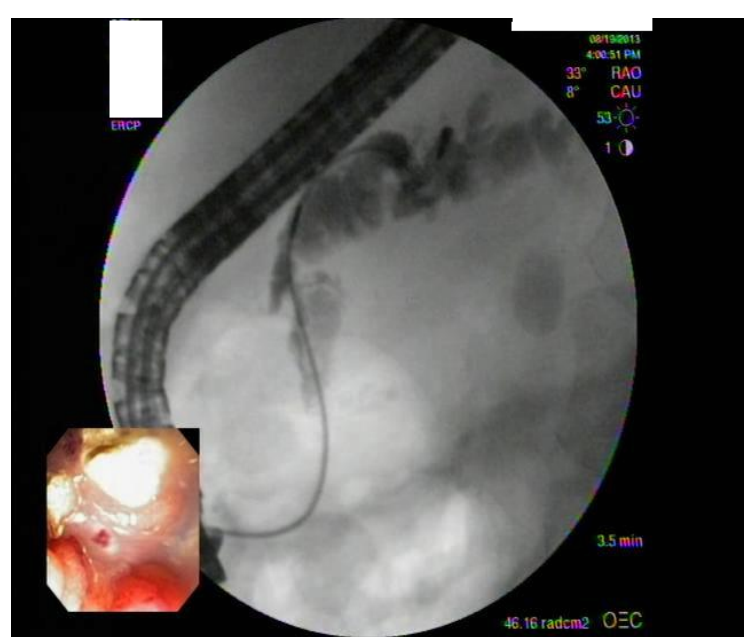

Figure 1. Extensive pancreatic stone burden seen during initial ERCP. Note the appearance of the ovoid right renal stone lateral to the lumbar vertebrae. Endoscopic view demonstrates stones within the pancreatic duct.

Prior to ESWL treatment, the patient had a computed tomography (CT) scan revealing diffuse dilatation of the pancreatic duct with multiple large pancreatic stones within the pancreatic body and tail. A $1.6-\mathrm{cm}$ renal stone, measuring 1480 Hounsfield units, was also identified at the right ureteropelvic junction (UPJ) with mild dilation of the proximal renal pelvis. However, given the patient's persistent epigastric abdominal pain and absence of urological symptoms, the Gastroenterology service decided to proceed with pancreatic lithotripsy with plans to refer the patient to urology for renal stone management at a later date.

Preoperative laboratory blood work revealed a hematocrit of 38.3 , platelet count of 200 , lipase of 57, and creatinine of 1.27. All coagulation profile values were found to be within normal ranges.

The patient underwent a pancreatic ESWL under general anesthesia using a Dornier Compact Delta II Lithotripter (electromagnetic) at an ambulatory surgical center that performs high-volume pancreatic lithotripsy. A scout image was initially taken with the patient in the supine position. Two radiopacities in the body and tail of the pancreas were identified and the patient was then placed in a right posterior oblique position (RPO) with the treatment head positioned presumably over the targeted stones (Figure 2).

A total of 2500 shocks were then administered using a power setting of 1 to 4 , ranging from 225 to 348 Bar. The treatment protocol consisted of an initial rate of 60 with 300 shocks administered. Voltage stepping was then used to complete the treatment. The procedure took a total of 40 minutes and fluoroscopy was performed afterwards with the finding of partial stone fragmentation.

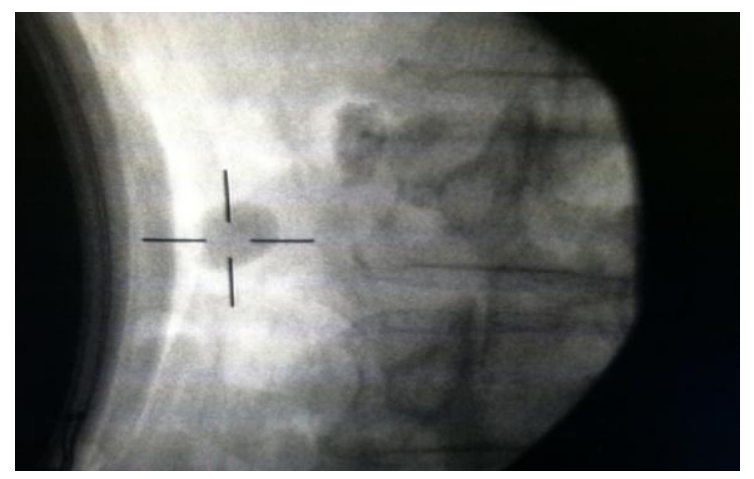

Figure 2. ESWL targeting of the perceived "pancreatic stone."

Shortly after the treatment, while still in the recovery room, the patient complained of rightsided flank pain with nausea and gross hematuria. After an unremarkable abdominal radiograph with normal blood work and an eventual improved in symptoms following hydration and supportive care, the patient was discharged home and instructed to return for follow-up in 2-3 weeks for repeat ERCP.

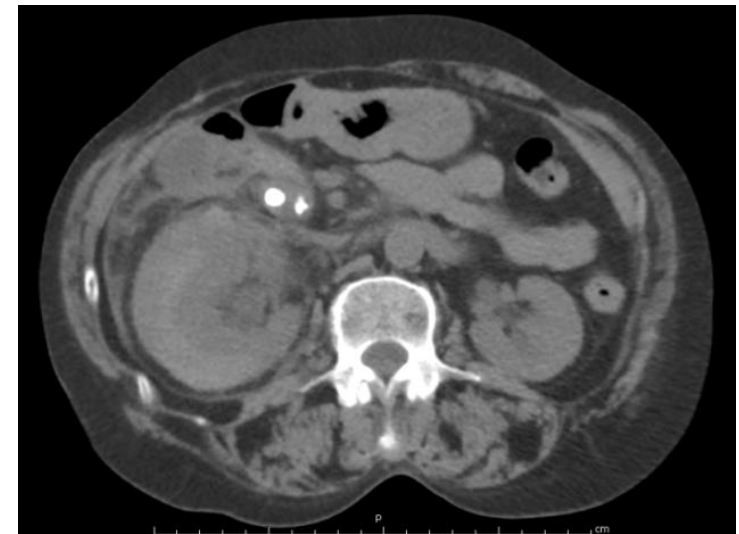

Figure 3. Post- ESWL CT scan revealing a moderate right-sided perinephric hematoma as well as the posterior spatial relationship of the right kidney to the pancreatic stone burden.

Three days later, however, the patient presented to the hospital emergency room with worsening right-sided flank pain with nausea and a lowgrade fever. Laboratory blood work revealed a hematocrit of 29.5, platelet count of 145, lipase of 38, and an elevated creatinine of 2.38. A CT scan without contrast was obtained revealing a moderate-sized right renal perinephric and subcapsular hematoma (Figure 3) with moderate hydronephrosis secondary to a $1.2-\mathrm{cm}$ calculus at the right UPJ (Figure 4). Multiple other smaller stone fragments were also seen scattered 
within the renal pelvis and no interval changes were noted within the pancreatic stone burden. Urology consultation was then requested.

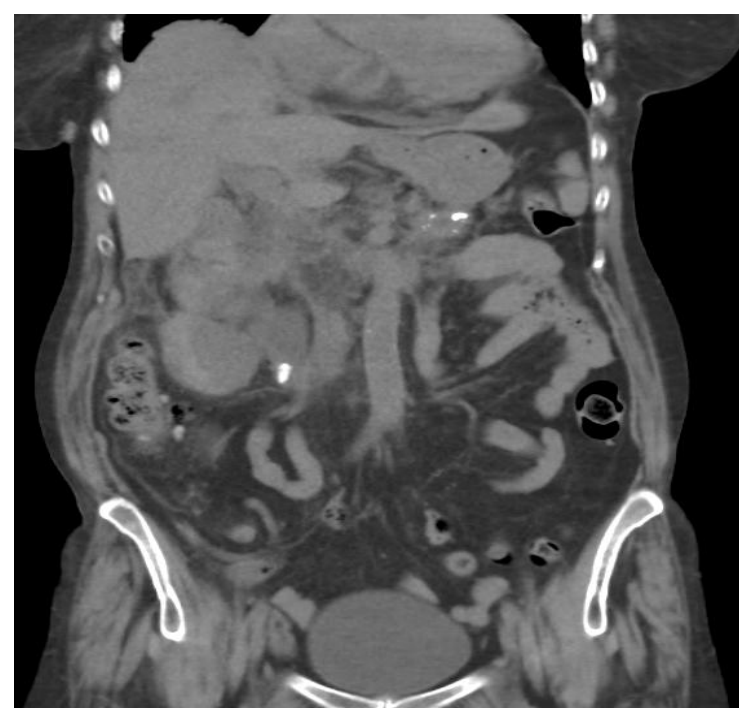

Figure 4. Post- ESWL CT Scan revealing significant hydronephrosis secondary to an obstructing stone at the level of the right UPJ.

Due to the patient's elevated creatinine, which was felt to be due to an obstructing UPJ stone in the setting of a perinephric hematoma, the decision was made to bring the patient to the operating room emergently for placement of an indwelling ureteral stent. A $6 \times 24 \mathrm{~cm}$ pigtail stent was subsequently placed without any difficulties.

Over the next few days, the patient was managed conservatively with initial bed rest and serial hematocrit levels. One unit of packed red blood cells was transfused following a drop in hematocrit to 22.4 in the setting of worsening tachycardia. Additionally, ultrasound imaging was serially performed to ensure the stability of both perinephric and subcapsular hematomas. No evidence of hematoma expansion was evident on repeat ultrasound and after several days both hematomas were found to be stable in size with marked improvement of the previous right-sided hydronephrosis.

The patient was ultimately discharged home following 9-days of hospitalization. At the time of discharge, both hematocrit and creatinine levels had returned to near-baseline levels. A repeat CT scan performed 3-months following the initial presentation demonstrated complete resolution of both perinephric and subcapsular hematomas (Figure 5). The patient subsequently underwent an uncomplicated ureteroscopy with laser lithotripsy of the right renal stones and was rendered stone-free at the conclusion of treatment. Crystallographic analysis demonstrated the stones to be calcium oxalate dihydrate.

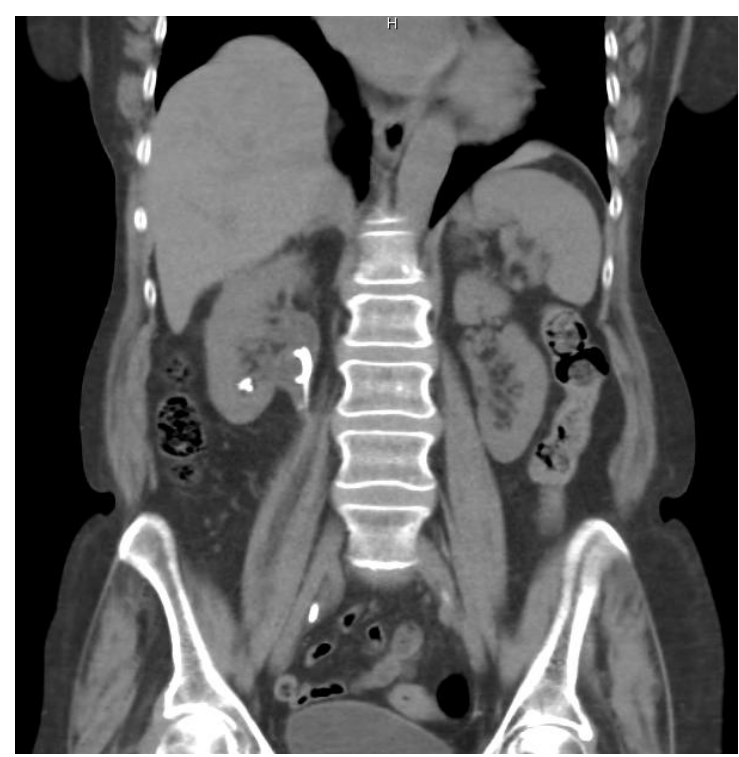

Figure 5.CT scan revealing resolution of perinephric and subcapsular hematoma with interval resolution of hydronephrosis following ureteral stenting.

\section{DISCUSSION}

Collateral tissue injury is a rare but welldocumented complication following ESWL therapy. In the urologic literature, multiple cases reports have described injuries to neighboring organ systems resulting either directly from parenchymal damage by dissipated shockwaves or indirectly from stone fragmentation following ESWL therapy for renal and ureteral stones [69]. Similar complications have resulted following pancreatic ESWL [3-5]. However, the finding of a perinephric hematoma with resulting AKI following a pancreatic lithotripsy is the first to demonstrate an important and potential danger of ESWL, the mistargeting of the intended stone due to adjacent organ stone disease.

Pre-existing hydronephrosis has not been identified as a risk factor for the development of acute renal side effects after ESWL. Higher hematoma rates between $3-12 \%$ have been reported in later generations of lithotripters, presumably related to the higher peak pressures that are generated with a smaller focal area [10]. Advanced age has also been identified as a factor that increases the likelihood of subcapsular hematoma with a 2.2-fold increase for every 10 year increase in the patients age[6].

As previously mentioned, prior to pancreatic lithotripsy, the patient was noted to have a large obstructing renal stone at the right UPJ with 
resulting hydronephrosis. Although asymptomatic from this renal pathology, the patient denied any prior right flank pain and showed no clinical evidence of renal deterioration. As a result, a pre-operative urological consult was delayed in favor of pancreatic lithotripsy due to the patient's persisting epigastric pain. The delay in requesting a timely evaluation by a urologist in this case proved to be a critical oversight that contributed to the resulting complications that this patient experienced.

Upon initial urological consultation and review of the patient's CT scan following ESWL (Figures 3and 4), the question of inadvertent localization of the pancreatic stones with the patient's known history of a renal stone was not raised. Plain films taken during the time of ESWL were not available and, given the high volume and prior outcomes of the lithotripsy center, it was believed that the fragmentation of the $1.6 \mathrm{~cm}$ right renal stone and subsequent hematoma were the result of collateral injury sustained indirectly during lithotripsy of the pancreas. However, after obtaining access to the imaging records from prior ERCP as well as the intraoperative films, it became apparent that an error in lithotripter stone targeting likely occurred and the targeted pancreatic stone was actually the pre-existing renal stone.

Figure I, which was obtained at the time of ERCP and which pre-dates the ESWL, demonstrates the endoscope positioned with a basket in the pancreatic duct. In this image, an ovoid stone is apparent inferior to the pancreas and represents the untreated $1.6-\mathrm{cm}$ right renal stone. The radiograph in Figure 2, taken at the time of the pancreatic ESWL, demonstrates the cross-hair target positioned on the ovoid stone which is not the intended pancreatic stone but rather the previously untreated renal stone. This error in localizing the correct stone resulted in the partial fragmentation of the right renal stone and led to hematoma formation.

As with renal or ureteral lithotripsy, stone targeting and accurate localization for pancreatic lithotripsy is accomplished by reviewing prior radiographic images of the stones and by obtaining good quality plain films with the patient properly positioned during time of lithotripsy. For pancreatic ESWL, patients are generally placed in the RPO position with an angled wedge in order to isolate the pancreas from other vital structures as best as possible. General anesthesia is often administered to control respiratory rate and reduce potential stone motion. Shockwaves are then delivered from above in a direct line with the shortest path to the stone. These measures, however, may not be enough to overcome errors in the initial stone targeting when there is concomitant renal stone disease.

Patients who have stone disease in the pancreas and the kidney present a unique challenge for both the gastroenterologist and urologist. Without definitive satisfactory imaging of the intended pancreatic target, cases such as this should not proceed until proper imaging is obtained. However, even with appropriate imaging, an intraoperative urology consult could be obtained to ensure proper stone targeting. Moreover, a simple on-table injection with IV contrast can better define the urinary tract and allow for more precise pancreatic targeting that will result in better fragmentation and a safer outcome for the patient.

\section{COnClusions}

As this case clearly demonstrates, it is imperative that all clinicians involved in ESWL, urologists, gastroenterologists, as well as lithotripter technicians, become aware of this potential injury and collaborate to prevent this from occurring. In cases of coexisting renal and pancreatic stones, we believe that any obstructive renal stones should be treated prior to pancreatic ESWL. In cases of small and nonobstructing renal stones, preoperative urologic consultation should still be obtained but the management for the urinary stones may be deferred. In this setting, the use of intravenous contrast may be sufficient to delineate the upper urinary tract and allow more definitive stone targeting of the intended pancreatic stone.

\section{REFERENCES}

[1] Sauerbruch .T, Holl J., Sackmann M., et al. Disintegration of a pancreatic duct stone with extracorporeal shock waves in a patient with chronic pancreatitis, Endoscopy. 19 (1987).

[2] Tandan M. and Reddy D.N. Extracorporeal shock wave lithotripsy for pancreatic and large common bile duct stones, World J Gastorenterol. 17 (2011).

[3] Kazuo I., et al. Treatment of pancreatic stones with extracorporeal shock wave lithotripsy: results of a mulitcenter survey, Pancreas. 30 (2005).

[4] Hirata N., Kushida Y., Ohguri T., Wakasugi S., Kokima T., Fujita R. Hepatic subcapsular hematoma after extracorporeal shock wave 
lithotripsy (ESWL) for pancreatic stones, J Gastroenterol. 34 (1999).

[5] Leifsson B.G., Borgstrom A., Ahlgren G. Splenic rupture following ESWL for a pancreatic duct calculus, Dig Surg. 18 (2001).

[6] Dhar N.B., Thornton J., Karafa M.T., Streem S.B. A multivariate analysis of risk factors associated with subcapsular hematoma formation following electromagnetic shockwave lithotripsy, J Urol. 172 (2004).

[7] Kim T.B., Park H.K., Lee K.Y., et al. Lifethreatening complication after extracorporeal shock wave lithotripsy for a renal stone: a hepatic subcapsular hematoma, Korean J Urol. 51 (2010).

[8] Hassan I. and Zietlow S.P. Acute pancreatitis after extracorporeal shockwave lithotripsy for a renal calculus, Urology. 60 (2002).

[9] Hung S., Chen H., Jan Y., et al. Common bile duct and pancreatic injury after extracorporeal shock wave lithotripsy for renal stone, Hepatogastroenterology. 34 (2000).

[10] Piper N.Y., Dalrymple N., Bishoff J.T. Incidence of renal hematoma formation after ESWL using the new Dornier Doli-S lithotripter, J Urol, 165 (2001).

Citation: Nitin Sharma \& Jay A. Motola. Pancreatic Stone Extracorporeal Shockwave Lithotripsy-A New Concern for Urologists?.ARC Journal of Urology.2018; 3(1):1-5. doi:dx.doi.org/10.20431/2456-060X.03 01001.

Copyright: (C) 2018 Authors. This is an open-access article distributed under the terms of the Creative Commons Attribution License, which permits unrestricted use, distribution, and reproduction in any medium, provided the original author and source are credited. 